\title{
A METASTATIC DEPOSIT IN THE PATELLA FROM A CARCINOMA OF THE BREAST
}

\author{
Leslie Klenerman, M.B., F.R.C.S., \\ Senior Registrar to the Orthopadic Department, The Middlesex Hospital, London, W.1.
}

Primary tumours of the patella are rare. Osteoclastoma is the commonest neoplasm and twenty cases have been reported (Goodwin, 1961). Secondary involvement by tumour is most uncommon and may result either from direct spread by a primary sarcoma or a metastasis in the region of the knee joint, or from metastasis of a distant tumour. Thus two cases of metastasis from prostatic carcinoma have been recorded (Schmidt, 1907; Schwartz, 1909; both quoted by Cole, 1925). A pathological fracture occurring through a patella infiltrated by secondary tumour must be extremely unusual.

\section{Case Report}

The patient was a housewife aged forty-eight years. She was referred to the surgical out-patient department for a lump in her right breast which she had noticed three months previously. In September, 1959 a right radical mastectomy was performed. Histological examination showed a spheroidal cell carcinoma. One of eleven axillary lymph nodes sectioned contained growth. After operation the patient was treated by deep X-ray therapy and 4,500 rads. were given to the internal mammary and supraclavicular regions.

She remained well for four years until in September, 1963 signs of superior vena caval obstruction due to mediastinal lymph node involvement developed. Radiotherapy was given to the mediastinum (1,330 rads.) and the patient given Prednisone, $15 \mathrm{mg}$. daily.

In June, 1964 she complained of pain in the lower part of her back. Radiographs revealed osteolytic deposits in the left ilium and sacroiliac region. Deep X-ray therapy to this area was commenced. While going downstairs after treatment in the Radiotherapy Department, she suddenly felt her left knee give way and acute pain in the joint developed.

On Examination. There was a moderate effusion in the left knee. Flexion was limited by about thirty degrees due to pain. Radiographs showed an area of trabecular destruction in the proximal two-thirds of the patella and there was a transverse crack through the middle of this area with minimal displacement. The adjacent femur and tibia were normal (Fig. 1).

Treatment. The patella was enucleated through a vertical skin incision. Routine physiotherapy was started on the first day. On the twelfth day an effusion was aspirated. Three weeks after operation the patient was able to go home walking with a stick. Arrangements made for her review could not be carried out as her general condition deteriorated

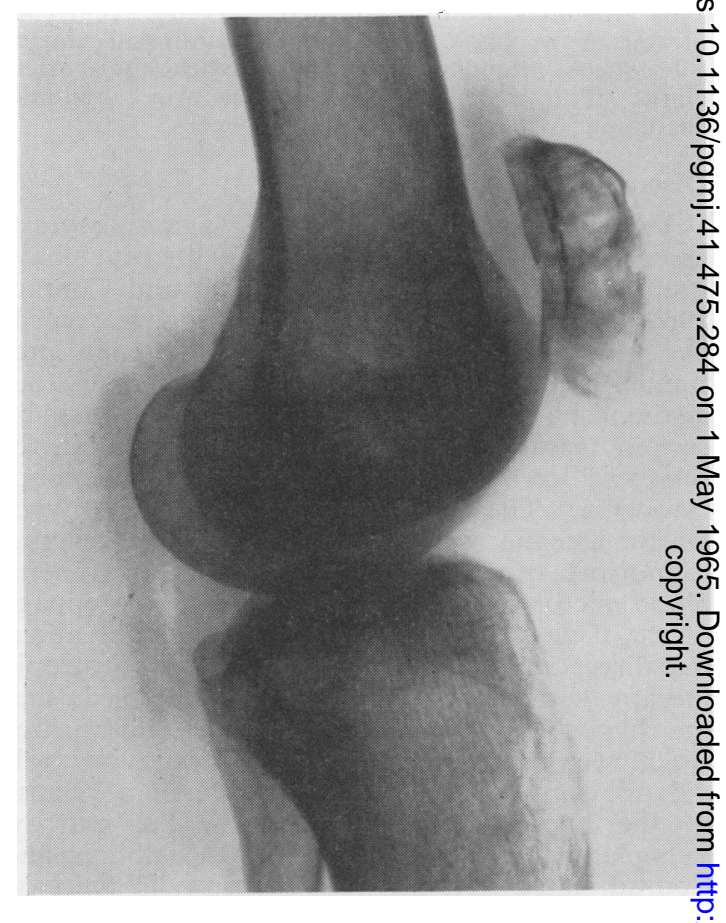

Fig. 1.-The lateral radiograph of the patella, showit a crack fracture through an osteolytic area the proximal two-thirds.

rapidly and she died three months after the operation.

Pathology. The articular surface of the patelfa was smooth but the fracture site was clearly visibfe. The subcutaneous surface was somewhat hæmorrhagic but quite intact (Fig. 2). Histological examination showed spheroidal cells similar to those in the primary breast carcinoma (Figs. 3, 4).

\section{Discussion}

Breast carcinoma frequently spreads to bote and in some clinics an incidence as high os seventy-eight per cent has been recorde्ed (Copeland, 1964). Bones in the distal segments :of limbs are rarely affected unless metastases ape widespread. Deposits tend to occur chiefly 9 t the sites of red bone marrow, for exampRe, vertebrae, pelvis, ribs, proximal ends of femota 


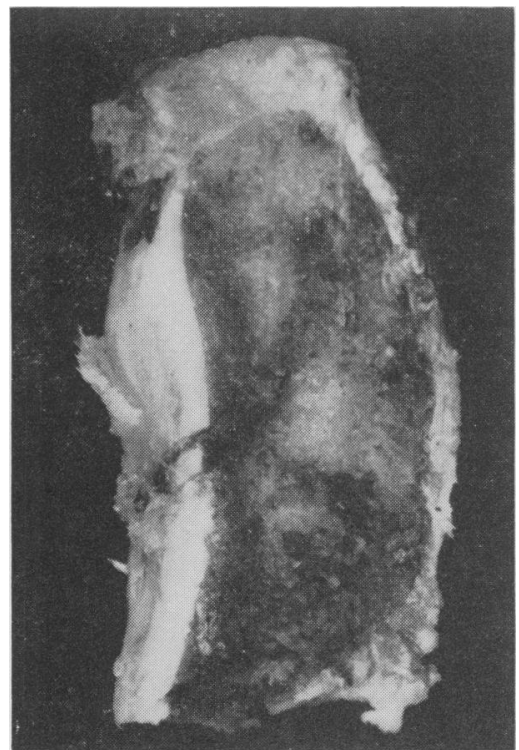

FIG. 2.-Photograph of the patella (x2). The fracture line through the articular cartilage is clearly seen.

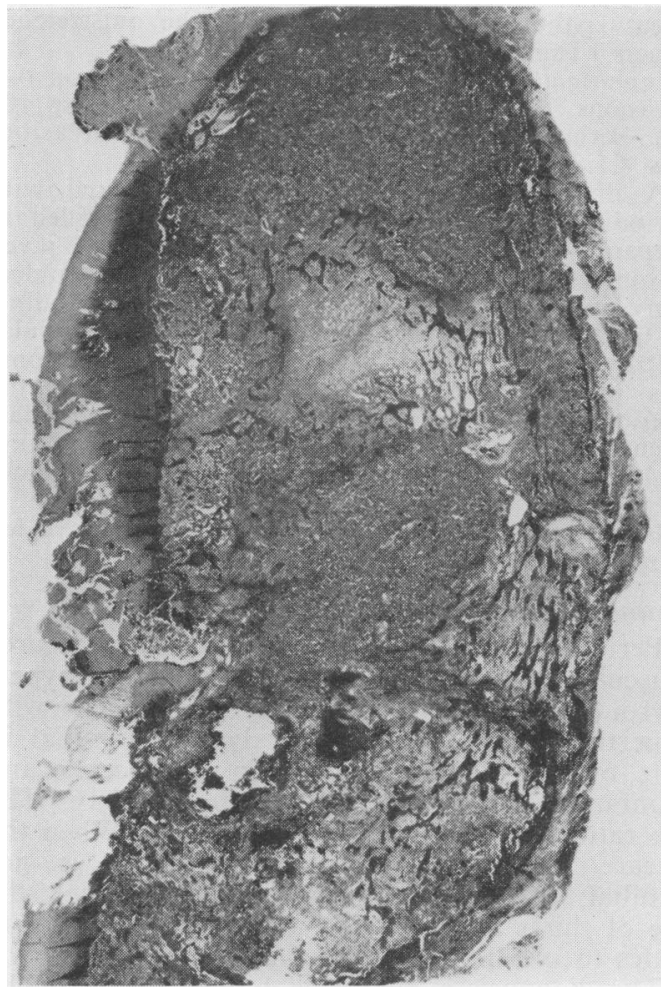

FIG. 3.-Photomicrograph (x2) showing cells replacing the cancellous bone of the upper two-thirds of the patella.

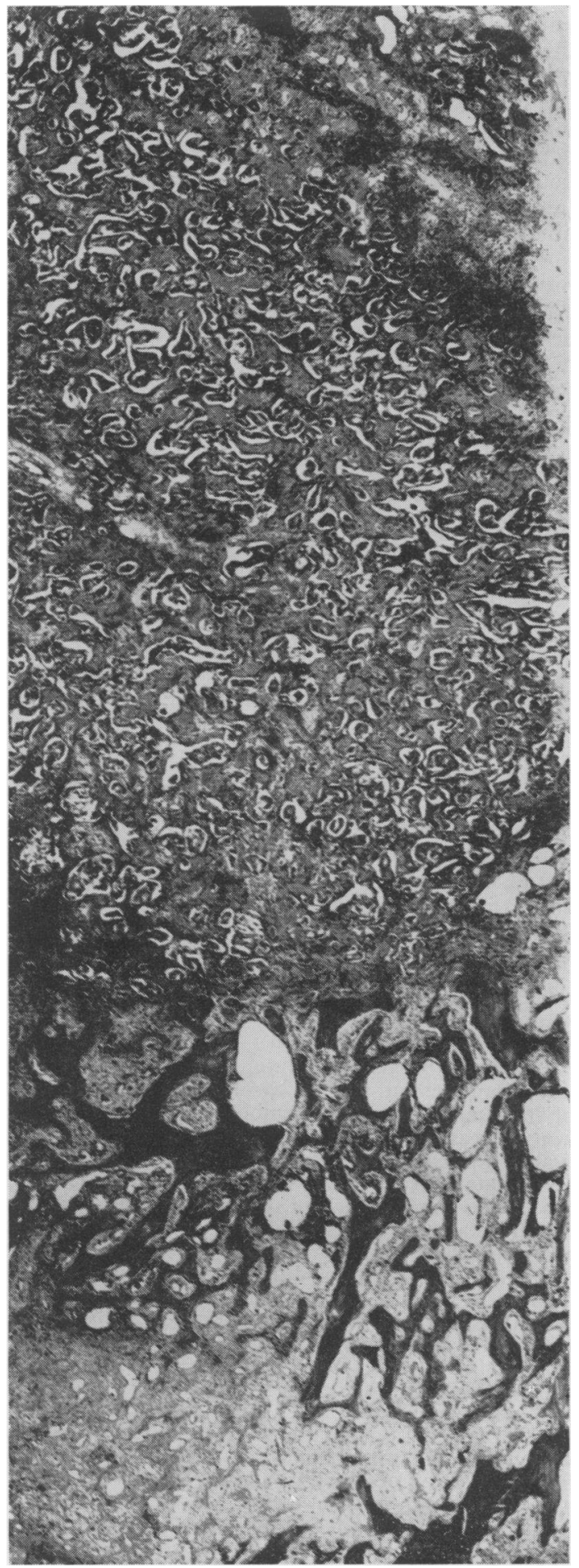

FIG. 4.-Photomicrograph (x40) showing tumour cells filling the centre of the field with areas of normal bone on either side. 
and humeri. It is therefore difficult to account for the occasional involvement of bones such as the patella, clavicle and scapula. The small size of the patella and its dense cancellous structure make the bone an unlikely site for a metastatic deposit. Joll (1923) suggested that trauma may determine the establishment of a circulating tumour embolus. In this case, however, there was no previous history of injury.

\section{Summary}

A pathological fracture through a secondary deposit of breast carcinoma in a patella is described. As far as can be ascertained, no previous case has been recorded.
I would like to thank Miss M. D. Snelling for permission to record this case, and also Dr. P. Byers and the Photographic Department of the Institud of Orthopaedics for their help.

\section{REFERENCES}

Cole, W. H. (1925): Primary Tumours of these Patella, J. Bone Jt. Surg., 7, 637.

COPELAND, M. M. (1964): Treatment of Cancer an Allied Diseases. Edited by George T. Pack and Irving M. Ariel, p. 502. New York: Hoeber.

GoodwIN, M. A. (1961): Primary Osteosarcoma of the Patella, J. Bone Jt. Surg., 43B, 338.

Joll, C. A. (1923): Metastatic Tumours of Bone Brit. J. Surg., ii, 38.

\title{
HYPERTHYROIDISM COMPLICATED BY
}

\section{PERIODIC PARALYSIS}

\author{
J. A. DyDE, F.R.C.S., \\ Surgical Registrar, The Royal Hospital, Sheffield.
}

\begin{abstract}
Periodic paralysis associated with hyperthyroidism is extremely rare outside Japan. In a series of 6,333 cases of hyperthyroidism admitted to three hospitals in Japan the incidence was found to be $2 \%$ (Okinaka, 1957). Twenty cases have been reported in America (Bartels and Pouget, 1963) and four cases have been reported in England (Petch, 1964). A further case is reported below.
\end{abstract}

\section{Case Report}

H. B., a 32-year-old fitter, who had previously been in excellent health, awoke in the early hours of the morning of December 27th, 1963 to find himself paralysed from the waist down. His lower limbs gradually regained their full strength five hours later. He had no more trouble until January 9th when he awoke in the early hours of the morning to find himself unable to move his arms and legs, but able to breathe, speak and swallow normally. He had no difficulty in controlling his bladder or bowels. During this second attack he was examined by his practitioner who found that he had a flaccid paralysis with normal sensation and absent limb reflexes. This attack lasted nine hours. On several occasions after these two attacks his thighs felt weak and heavy after he had been sitting for some time, but this sensation wore off after he had walked around the room for a while. There was no family history of similar attacks.

A few weeks before his first attack of paralysis he noted that he had become severely irritable and, despite a very good appetite, he had lost two stones in weight.

On Examination he had no abnormal neurological signs, but he had a moderate-sized nodular goitre. His pulse rate was 112 per minute, he had hot sweaty palms and a fine tremor of the outstretofes
fingers. There were no eye signs.

Investigations. BMR $+92 \%$ and $+75 \%$, on occasions. Serum protein bound iodine $12.4 \mu \mathrm{g} / 00$ ml., serum potassium on four separate occasions was $4.1,4.4,4.0,3.3 \mathrm{mEq} . / 1$.

A diagnosis of hyperthyroidism associated with periodic paralysis was made and it was decided to prepare him for surgery. First he was givel carbimazole. Ten days before sub-total thyroides tomy was performed he was given Lugol's Iodine

Operation. The clinical impression of a nodulat goitre was confirmed and sub-total thyroidectom was performed. The patient's recovery from the operation was straightforward and when he laș attended out-patients six months later he was found. to be euthyroid and he had had no more attacks of paralysis.

Histological examination of the thyroid revealed a hyperplastic gland with scanty colloid.

\section{Comment}

Periodic paralysis is the rarest of the neuro muscular disorders which can complicate hypero thyroidism. When it is not associated wit hyperthyroidism, periodic paralysis is familial in $80 \%$ of cases, occurs usually in adolescence and spontaneously regresses at the age of thirty. The sex ratio is three males to one female. When the disease complicates hyperthyroidism it is not familial; it most commonly occurs between the age of thirty and fifty and the sex ratio is twent males to one female (Okinaka, 1957).

The diagnosis is suggested by the history. The attacks usually occur in the early hours of the morning and last several hours before ceasing spontaneously. The muscles of the limbs are 